\title{
Information Tools for Special Examination Analysis of the Anthropogenic Impact on Plant Ranges Using Remote Sensing Data
}

\author{
Victor P. Savorskiy ${ }^{1}$, Alexandr V. Kashnitskii ${ }^{2}$, Olga Yu. Panova ${ }^{1}$, Evgeny V. Savchenko ${ }^{1}$ \\ ${ }^{1}$ Kotelnikov Institute of Radioengineering and Electronics of Russian Academy of Sciences, Fryazino \\ Branch, Fryazino, Russia \\ savor@inbox.ru \\ ${ }^{2}$ Space Research Institute of Russian Academy of Sciences, Moscow, Russia \\ kashnizky@gmail.com
}

\begin{abstract}
The work is devoted to the development of methods for controlling the anthropogenic impact on plant ranges. Elaborated methodical approach is implemented in the form of software products, namely web tools of VEGAScience service, which have to ensure the fulfillment of the target requirements necessary for identification of areas of anthropogenic impact by means of satellite monitoring. In this study, such a set of tools was applied to identify technical hemp crops, which is a model culture that has identical spectral signatures with narcotic cannabis. The developed tools made it possible to investigate and substantiate according real satellite observations the use of spectral indices for the sustainable identification of technical hemp fields against the background of spring wheat sowing.
\end{abstract}

Keywords: satellite monitoring, illegal cultivation of plants, spectral indices, Jeffreys-Matsushita distance 


\title{
ИНФОРМАЦИОННЫЕ ИНСТРУМЕНТЫ АНАЛИЗА АНТРОПОГЕННОГО ВОЗДЕЙСТВИЯ НА АРЕАЛЫ РАСТЕНИЙ ПО ДАННЫМ ДИСТАНЦИОННОГО ЗОНДИРОВАНИЯ ПРИ ПРОВЕДЕНИИ СПЕЦИАЛЬНЫХ ЭКСПЕРТИЗ
}

\author{
В.П. Саворский ${ }^{1}$, А.В. Кашницкийㄹ, О.Ю. Панова ${ }^{1}$, Савченко Е.В. ${ }^{1}$ \\ ${ }^{1}$ Институт радиотехники и электроники им. В.А. Котельникова РАН, Фрязинский филиал, \\ Фрязино, Московская обл., 141190 Россия \\ savor@inbox.ru \\ ${ }^{2}$ Институт космических исследований РАН, Москва, Россия \\ kashnizky@gmail.com
}

\begin{abstract}
Работа посвящена разработке методов контроля антропогенного воздействия на ареалы растений. Разработанный методический подход реализован в виде программных продуктов, web-инструментов сервиса «ВЕГА-Science», которые должны обеспечить выполнение требований к выявлению участков антропогенного воздействия по данным спутникового мониторинга. В исследовании такой набор инструментов применен для обнаружения посевов технической конопли, которая является модельной культурой, имеющей идентичные спектральные сигнатуры с наркотикосодержащей коноплей. Разработанные инструменты дали возможность исследовать и обосновать по результатам реальных спутниковых дистанционных наблюдений сельскохозяйственных угодий Центрально-европейского региона России в течение большей части вегетационного периода применение спектральных индексов для устойчивого выделения полей технической конопли на фоне посевов яровой пшеницы.
\end{abstract}

Ключевые слова: спутниковый мониторинг, незаконное культивирование растений, спектральные индексы, расстояние Джеффриса-Мацуситы

\section{Введение}

Одной из задач, существенно повышающих эффективность спутниковых методов наблюдения, является разработка специализированных информационных инструментов, обеспечивающих пользователей средствами выявления фактов незаконного культивирования растений по данным ДЗ3. Данная работа посвящена разработке такого рода информационных инструментов в составе крупной системы мониторинга, поддерживающей сервисы удаленного доступа к информационным ресурсам и средствам анализа хранимых в ней данных. Разработка этих инструментов основывается на требованиях [1], объективно обусловленных особенностями методов выявления участков незаконного культивирования растений средствами спутникового мониторинга. Разработанный в работе набор инструментов применен к исследованию участков посевов технической конопли средствами спутникового мониторинга, являющейся модельной культурой для исследования спектральных сигнатур участков наркотикосодержащей конопли [2]. Важной методической особенностью развиваемого в работе подхода является ориентация на исследование и разработку специализированных (или ориентированных) спектральных индексов, позволяющих, при прочих равных условиях, более надежно выявлять участки земной поверхности с запрещенными для выращивания культурами на фоне типовых для контролируемого региона сельскохозяйственных угодий. 


\section{1. Требования к информационным системам Д33, обеспечивающим решение задач выявления и оценки продуктивности результатов незаконного землепользования}

Исходя из результатов, полученных в рамках аналитического обзора [1], требования к сервисам информационных систем заключаются в необходимости обеспечивать решение задач выявления и оценки продуктивности результатов незаконного землепользования, включая обеспечение

1) возможностей автоматизированного совмещение разнородных и разновременных, включая архивные исторические, данных ДЗ3 и продуктов их обработки по регионам сельскохозяйственного производства с возможными участками незаконного землепользования;

2) возможностей автоматизированного совмещения данных и продуктов Д3З по регионам сельскохозяйственного производства с возможными участками незаконного землепользования с данными наземных наблюдений;

3) возможностей сбора данных и проведения их анализа для мониторинга фенологических фаз как контрольных растений, так и фоновых растительных покровов;

4) автоматизированного доступа к базам данных, описывающих физико-географические характеристике региона (в т.ч. топографию местности), особенности почвенного покрова и водного режима;

5) автоматизированного доступа к базам данных, описывающих климатические особенности (среднестатистические значения гидрометеорологических параметров и их среднеквадратические вариации);

6) доступа к оперативным данным и краткосрочным и среднесрочным гидрометеорологическим прогнозам (текущие показатели, синоптическая обстановка и накопленные значения почвенной влаги и снежного покрова);

7) доступа к описаниям типовых размеров участков и типовых агротехнических приемов в контрольном регионе (с учетом уклада и традиций местного населения);

8) доступа к описанию особенностей севооборота, в том числе частоты и особенностей применения смешанных посевов.

Указанные требования в значительной части могут быть выполнены с помощью информационного сервиса коллективного пользования «ВЕГА-Science» (http://sci-vega.ru/). В нем на базе ресурсов Центра коллективного пользования «ИКИ-Мониторинг» [3] организован доступ к долговременным большим архивам спутниковых данных. Имеющиеся в сервисе возможности работы с данными Д33 решают указанные выше требования по пп.1-4. Так как в сервисе одновременно со спутниковыми данными предоставляется доступ к метеоданным (NCEP Climate Forecast System Version 2, набор ds094.0, https://rda.ucar.edu/datasets/ds094.0), то требования по пп. 5,6 также выполняются при решении с помощью сервиса «ВЕГА-Science» задач выявления и оценки продуктивности результатов незаконного землепользования.

\section{2. Средства анализа результатов антропогенного воздействия на ареалы растений, предоставляемые сервисом «ВЕГА-Science»}

Для разработки устойчивой и стабильной методики выявления и оценки продуктивности результатов незаконного землепользования использовался сервис «ВЕГА-Science». Для решения задач настоящей работы важно то, что он предоставляет доступ к безоблачным наблюдениям целевых регионов в заданном периоде фенологического цикла. В частности, в нем возможно искать и обрабатывать архивные наборы данных дистанционных наблюдений с космического аппарата (KA) «Landsat-7» за период с 1999 года для средней полосы Европейской части России, включающие поля технической конопли. Также в нем доступны различные карты типов сельскохозяйственной растительности, покрывающие целевой регион. Такие карты регулярно строятся и обновляются в автоматическом режиме по данным прибора MODIS [4,5]. Для точного 
позиционирования и выделения постоянных объектов также возможно использовать высокодетальную подложку от компании DIGITAL GLOBE (http://mapsapi.digitalglobe.com). Таким образом, с помощью указанных здесь данных, в сервисе имеется необходимая возможность поиска и выбора полей $\mathrm{c}$ технической коноплей и полей с другими сельскохозяйственными культурами. Примененный в работе метод отбора таких полей будет описан далее.

Кроме доступа к долговременным архивам спутниковых данных, сервис «ВЕГА-Science» также поддерживает их углубленный анализ. Эта возможность предоставляется в виде набора информационных web-инструментов, которые позволяют выполнять обработку доступной в системе информации Д3З на мощностях самих центров хранения данных [6,7]. Такая обработка проводится в автоматическом режиме и описана в работах [8,9]. Получаемая при этом информация хорошо подходит для изучения особенностей землепользования на объектах большого размера, но не годится для целевых задач данной работы. Поскольку размеры исследуемых участков с культивированием незаконных растений невелики (типовые размеры составляют 300-500 м), в настоящей работе для выявления указанных участков применены результаты спутниковых наблюдений КА Landsat-7 с пространственным разрешением в требуемых спектральных каналах 30 метров на пиксель. При этом одним из основных критериев выбора контрольного участка было наличие обоснованного подтверждения вида выращиваемой на этом участке культуры.

Для анализа, заключающегося в выявлении изменений растительного покрова по данным дистанционных спутниковых наблюдений, состав и возможности информационных инструментов в сервисе «ВЕГА-Science» были расширены. Прежде всего, это вызвано необходимостью создания средств, которые в автоматическом или полуавтоматическом режиме позволили бы получить оценки статистик спектральных характеристик для отдельных полей или участков этих полей с визуально однородной растительностью. Для целей данной работы такими статистиками являются минимальное, максимальное и среднее значения, общее число пикселей на поле, дисперсия, коэффициент вариации. Важно отметить, что разработанные инструменты анализа позволяют оценивать не только статистические характеристики данных исходных дистанционных наблюдений и определяемые по ним коэффициенты отражения (на верхней границы атмосферы), но и статистики получаемых по ним спектральных индексов. При этом расширенный состав инструментов сервиса дает возможность проводить статистические оценки не только общепринятых индексов (например, NDVI), введенных заранее в сервис, но и индексов, формируемых пользователями в интерактивном режиме.

Получаемый в результате набор статистик дает возможность объективно оценивать различия индексов пар наблюдаемых полей в рамках выбранной метрики. В настоящей работе для этих целей нами используется расстояние Джеффриса — Матуситы $/ M$ [10]

$$
J M_{12}=2 \times\left(1-e^{-D_{12}}\right),
$$

которое в предположении нормальных распределений случайных величин оценивается через расстояние Бхаттачарии $D_{12}[11]$

$$
D_{12}=\frac{1}{4} \ln \left(\frac{1}{4}\left(\frac{\sigma_{1}^{2}}{\sigma_{2}^{2}}+\frac{\sigma_{2}^{2}}{\sigma_{1}^{2}}+2\right)\right)+\frac{1}{4}\left(\frac{\left(M_{1}-M_{2}\right)^{2}}{\sigma_{1}^{2}+\sigma_{2}^{2}}\right),
$$

где $M_{1}$ и $M_{2}$ - средние значения, а $\sigma_{1}$ и $\sigma_{2}$ - среднеквадратические вариации спектральных характеристик на участках 1 и 2 , соответственно.

\section{3. Порядок обработки спутниковых данных}

В рамках данной исследовательской работы были исследованы посевы технической конопли и окружающих ее ареалов сельскохозяйственных культур и участков естественных лесов (дикоросов), типичных для средних широт Европейской части РФ, описанные ранее в работе 
[12]. Посевы технической конопли были выбраны нами в соответствии с подходом, предложенном в [12], как объект для моделирования спутниковых мульти- или гиперспектральных наблюдений посевов конопли в видимом и ближнем ИК диапазонах. Этот подход подтвержден результатами работы [2], в которой показано, что спектральные характеристики конопли не зависят от концентрации содержащихся в ней канабиоидов, А это означает, что техническая конопля может быть использована как модельная культура для исследования спектральных сигнатур участков наркотикосодержащей конопли.

Для указанных в работе [12] участков технической конопли в сервисе «ВЕГА-Science» были выбраны все безоблачные сцены летнего сезона 2002 года с KA «Landsat-7», прибора ЕTM+. При идентификации участков технической конопли были использованы эти спутниковые данные, описания и планы размещения участков технической конопли из работы [12], а также публичные кадастровые карты (https://egrp365.ru/map). C использованием всех этих данных были также выбраны и локализованы участки яровых зерновых культур, как типовых растительных покровов, на фоне которых необходимо исследовать возможности обнаружения участков технической конопли по данным спутниковых дистанционных наблюдений в типовых условиях черноземного региона Европейской части России (Орловской области). В итоге для совместного анализа были отобраны 7 полей технической конопли и 9 полей яровой пшеницы.

По всем отобранным снимкам для всех полей был рассчитаны указанные выше в разделе 3 статистики коэффициентов отражения исследуемых полей (без атмосферной коррекции) для

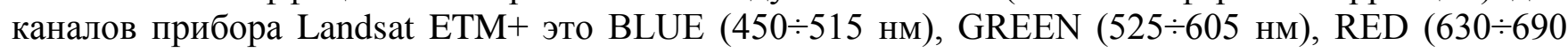
нм), NIR $\quad(775 \div 900 \quad$ нм $), \quad$ SWIR1 $(1550 \div 1750 \quad$ нм $), \quad$ SWIR2 $\quad(2080 \div 2350 \quad$ нм $)$ (https://landsat.gsfc.nasa.gov/the-enhanced-thematic-mapper-plus/). По выбранным сценам были рассчитаны спектральные индексы NDCI и NDVI и оценены статистики, описанные в выше разделе 3. Таким образом, была получена статистическая информация по 7 полям технической конопли и 9 полям яровых зерновых, т.е. фактически по 16 локальным сценам. Результаты интерпретации полученной информации представлены в следующем разделе.

\section{4. Результаты статистического анализа различимости полей технической конопли и яровых зерновых культур}

Для выбранных совокупности полей технической конопли и яровых зерновых культур в рамках анализа, базирующегося на web-инструментах ИС «ВЕГА-Science», были определены статистические характеристики их коэффициентов отражения (без атмосферной коррекции) на 3 срока вегетации 2002г. При этом было экспериментально подтверждено, что непосредственное использование отражательных характеристик не позволяет с высокой степенью достоверности различать поля полей технической конопли и яровых культур. Это проиллюстрировано на рис.1, где показано, что кластеры технической конопли и яровых в мае и августе для выбранных пар каналов ЕТМ+ практически не различимы.

Поэтому, в соответствии с общими рекомендациями [13], для повышения надежности обнаружения и контроля состояния целевых объектов наблюдения, полей технической конопли, в работе были использованы спектральные индексы. В частности, были исследованы статистические характеристики значения нормализованного дифференцированного индекса растительности NDVI [14]

$$
\mathrm{NDVI}=(\text { КСЯ канал4 }- \text { КСЯ канал3 }) /(\text { КСЯ канал4 }+ \text { КСЯ канал3 })
$$

и предложенного в [15] нормализованного вегетационного контрольного индекса (NDCI)

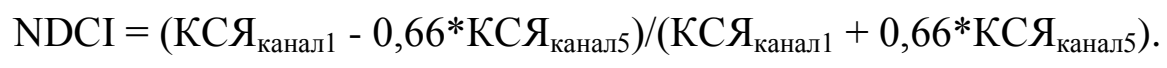




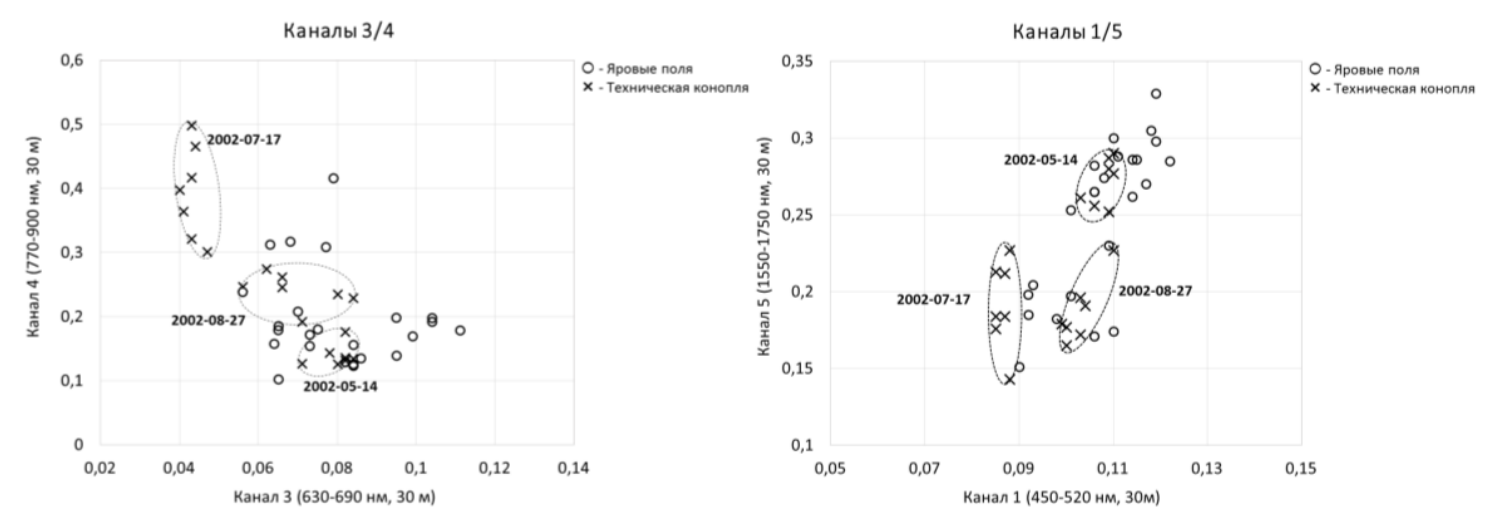

Puc.1. Диаграммы рассеяния средних значений КСЯ по отдельным сельскохозяйственным полям в 3 и 4 каналах; в 1 и 5 каналах ETM+ (Landsat-7) Орловская обл., 2002г.

В начале фенологического цикла посадки конопли и яровых культур практически не различимы и при использовании индексов. Но, как видно из рисунка 2, NDVI и NDCI, в целом, позволяют удовлетворительно различать поля технической конопли на фоне яровых вблизи максимума фенологического цикла (июль-август). При этом в указанный период наблюдается существенное различие в их динамике. Так, NDVI имеют за весь указанный период более высокие значения для конопли, чем для зерновых, а NDCI - меньшие значения в июле и большие в августе. Это свидетельствует о том, что эти индексы дополняют друг друга в описании фенологии этих культур, отражая то, что они регистрируют разные факторы, формирующие отражательные характеристики растительных покровов.
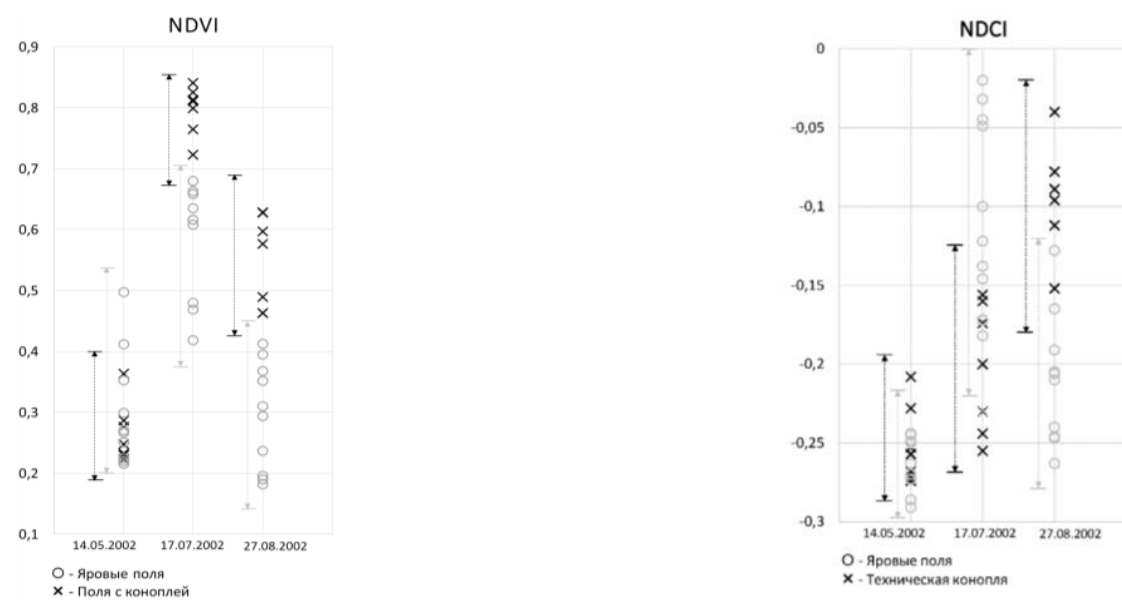

Puc. 2. Спектральные индексы полей технической конопли и яровых зерновых

a) NDVI, б) NDCI

Высокая надежность выделения полей технической конопли подтверждается и значениями расстояний Джеффриса-Мацуситы JM индексов NDVI и NDCI для пар полей технической конопли и яровой пшеницы, приведенными в таблице 1. Представленные в ней усредненные по всем возможным парам сочетаний пар полей технической конопли и яровой пшеницы расстояния JМ в большинстве случаев превышают 1,5. Это указывает на значительное различие средних статистических значений этих индексов для июля и августа. В этом случае вероятность пропуска участков конопли (по критерию JM $<1$ для NDVI или NDCI) не превышает 4\%. В мае расстояния JM для NDVI и NDCI в большинстве случаев не превышают 1, что объективно связано с состоянием растительного покрова.

В таблице 2 показаны усредненные расстояния JM индексов NDVI и NDCI для пар полей технической конопли. В этом случае вероятность пропуска участков конопли (по критерию JM > 
1 для NDVI и NDCI) ${ }^{1}$ не превышает 30\% для всей совокупности полей конопли и 14 \% при исключении из статистики поля «Брянцево-1», которое находилось в стадии вегетации, отличной от стадий вегетации всех прочих полей.

Таблица 1. Средние и среднеквадратические значения расстояний JM индексов NDVI/NDCI (усреднение по всей совокупности пар полей технической конопли и яровой пшеницы)

\begin{tabular}{|c|c|c|c|c|c|c|c|c|c|c|c|c|c|c|c|}
\hline \multirow{2}{*}{\multicolumn{2}{|c|}{ Сезон 2002Г }} & \multicolumn{2}{|c|}{ Болхов -1 } & \multicolumn{2}{|c|}{ Кромы-2 } & \multicolumn{2}{|c|}{ Кромы-1 } & \multicolumn{2}{|c|}{ Брянцево-1 } & \multicolumn{2}{|c|}{ Брянцево-2 } & \multicolumn{2}{|c|}{ Брянцево-3 } & \multicolumn{2}{|c|}{ Хотынец-1 } \\
\hline & & NDVI & $\mathrm{NDCI}$ & NDVI & NDCI & NDVI & $\mathrm{NDCI}$ & NDVI & NDCI & $\overline{\mathrm{NDVI}}$ & NDCI & NDVI & NDCI & NDVI & NDCI \\
\hline M & \multirow{2}{*}{14.05} & 0,73 & 0,91 & 1,09 & 1,41 & 0,95 & 0,55 & 1,00 & 0,71 & 1,05 & 0,72 & 1,02 & 0,48 & 0,57 & 0,33 \\
\hline$\sigma$ & & 0,86 & 0,60 & 0,67 & 0,44 & 0,62 & 0,50 & 0,70 & 0,18 & 0,72 & 0,23 & 0,73 & 0,25 & 0,51 & 0,33 \\
\hline $\mathrm{M}$ & \multirow{2}{*}{17.07} & 1,21 & 0,81 & 1,93 & 1,03 & 1,86 & 1,28 & 2,00 & 1,76 & 1,99 & 1,45 & 1,99 & 0,88 & 1,76 & 1,76 \\
\hline$\sigma$ & & 0,65 & 0,76 & 0,09 & 0,80 & 0,18 & 0,60 & 0,01 & 0,35 & 0,02 & 0,60 & 0,02 & 0,57 & 0,24 & 0,35 \\
\hline $\mathrm{M}$ & \multirow{2}{*}{27.08} & 1,52 & 1,06 & 1,95 & 1,93 & 1,64 & 1,77 & 1,98 & 1,85 & 1,95 & 1,89 & 1,72 & 1,87 & 1,91 & 1,64 \\
\hline$\sigma$ & & 0,68 & 0,69 & 0,10 & 0,14 & 0,59 & 0,34 & 0,04 & 0,25 & 0,10 & 0,19 & 0,48 & 0,23 & 0,16 & 0,46 \\
\hline
\end{tabular}

Таблица 2. Средние и среднеквадратические значения расстояний JM индексов NDVI/NDCI (усреднение по всей совокупности пар полей технической конопли 17.07.2002)

\begin{tabular}{|c|c|c|c|c|c|c|c|c|c|c|c|c|c|c|}
\hline \multirow[t]{2}{*}{ Сезон 2002г. } & \multicolumn{2}{|c|}{ Болхов -1 } & \multicolumn{2}{|c|}{ Кромы-2 } & \multicolumn{2}{|c|}{ Кромы-1 } & \multicolumn{2}{|c|}{ Брянцево-1 } & \multicolumn{2}{|c|}{ Брянцево-2 } & \multicolumn{2}{|c|}{ Брянцево-3 } & \multicolumn{2}{|c|}{ Хотынец-1 } \\
\hline & NDVI & NDCI & NDVI & NDCI & NDVI & NDCI & NDVI & NDCI & NDVI & NDCI & NDVI & NDCI & NDVI & NDCI \\
\hline & & & & & & & & & & & & & & \\
\hline & & & & & & & & & & & & & 29 & 52 \\
\hline & & & & & 00 & & & & & & & &, 71 & 00 \\
\hline & & & & & 96 & & & & & 98 & & & , 94 & 17 \\
\hline & 1,5 & 0,81 & & & 1,74 & 1,55 & & & & 00 & & & 0,67 & 35 \\
\hline & 1,4 & 0,2 & & 4 & 1,57 & 1,0 & & & & 0,32 & & & 0,80 & 0,87 \\
\hline 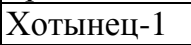 & 0,61 & 1,47 & 0,29 & 1,52 & 0,71 & 2,00 & 0,94 & 0,17 & 0,67 & 0,35 & 0,80 & 0,87 & 0,00 & 0,00 \\
\hline
\end{tabular}

\section{Заключение}

В работе описаны средства анализа спутниковых дистанционных наблюдений, применяемые для выявления незаконного культивирования растений как результатов антропогенного воздействия на ареалы растений. Эти средства реализованы в составе сервисов «ВЕГА-Science». Наряду с описанием порядка обработки спутниковых данных инструментами сервисов «ВЕГА-Science», показаны преимущества их применения для анализа спектральных индексов NDVI и NDCI. Эффективность применения индексов оценена по величинам расстояний Джеффриса-Мацуситы JM индексов NDVI и NDCI между полями целевой культуры (технической конопли) и контрольными полями (яровой пшеницы). Оценки вероятностей пропуска полей технической конопли подтверждают высокую эффективность развитого в работе подхода.

Исследование выполнено при поддержке РФФИ в рамках научного проекта № 16-2909615 офи_м.

\footnotetext{
${ }^{1}$ Отмечено в таблице 2 полужирным курсивом
} 


\section{References}

[1] Savorskiy V.P., Panova O.Yu., Savchenko E.V., Methods for analyzing satellite monitoring data on plant areals to identify illegal crop fields in the conduct of special examinations, Sovremennye problemy distantsionnogo zondirovaniya Zemli iz kosmosa, 2018a, 15(5), pp. 13-30. DOI: 10.21046/2070-7401-2018-15-513-30. (In Russian).

[2] Walthall C.L., Daughtry C. S.T., Pachepsky L., Development of Cannabis Spectral Signatures and Cannabis Growth Simulation Model: Progress report FY 2003. Beltsville, MD, USA: USDA-ARS Hydrology and Remote Sensing Laboratory, 2003, 15 p.

[3] Loupian E.A., Proshin A.A., Burtsev M.A., Balashov I.V., Bartalev S.A., Efremov V.Yu., Kashnitskiy A.V., Mazurov A.A., Matveev A.M., Sudneva O.A., Sychugov I.G., Tolpin V.A., Uvarov I.A., IKI center for collective use of satellite data archiving, processing and analysis systems aimed at solving the problems of environmental study and monitoring, Sovremennye problemy distantsionnogo zondirovaniya Zemli iz kosmosa, 2015, 12(5), pp. 263-284. (In Russian).

[4] Plotnikov D.E., Bartalev S.A., Lupyan E.A., Method of summer-fall sprouts of winter crops detection with MODIS radiometer data, Sovremennye problemy distantsionnogo zondirovaniya Zemli iz kosmosa, 2008, 5(2), pp. 322-330. (In Russian).

[5] Bartalev S.A., Egorov V.A., Loupian E.A., Plotnikov D.E., Uvarov I.A., Recognition of arable lands using multi-annual satellite data from spectroradiometer modis and locally adaptive supervised classification, Komp'yuternaya optika, 2011, 35(1), pp. 103-116. (In Russian).

[6] Kashnitskiy A.V., Balashov I.V., Loupian E.A., Tolpin V.A., Uvarov I.A., Development of software tools for satellite data remote processing in contemporary information systems, Sovremennye problemy distantsionnogo zondirovaniya Zemli iz kosmosa, 2015, 12(1), pp. 156-170. (In Russian).

[7] Kashnitskiy A.V., Loupian E.A., Balashov I.V., Konstantinova A.M., Technology for Designing Tools for the Process and Analysis of Data from Very Large Scale Distributed Satellite Archives, Optika atmosfery i okeana, 2016, 29(9), pp. 772-777. DOI: 10.15372/AOO20160908.

[8] Tolpin V.A., Bartalev S.A., Burtsev M.A., Efremov V.Yu., Loupian E.A., Mazurov A.A., Matveev A.M., Proshin A.A., Flitman E.V., Interannual dynamics based tstimation of crops conditions by MODIS data, Sovremennye problemy distantsionnogo zondirovaniya Zemli iz kosmosa, 2007, 4(2), pp. 380-389. (In Russian).

[9] Tolpin V.A., Loupian E.A., Bartalev S.A., Plotnikov D.E., Matveev A.M., Agricultural crops analysis abilities with the VEGA satellite service, Optika atmosfery i okeana, 2014, 27(7), pp. 581-586. (In Russian).

[10] Richards J.A., Jia X., Remote Sensing Digital Image Analysis, Berlin Heidelberg: Springer, 2006, 439 p. DOI: https://doi.org/10.1007/3-540-29711-1

[11] Chernyavsky G.M., Strykov A.I., System approach and new information technologies in the detection of narcotic plants, Moscow: ANO Nauchno-informatsionnyy izdatel'skiy tsentr, 2003, 207 p.

[12] Savorskiy V.P., Kashnitskiy A.V., Konstantinova A.M., Balashov I.V., Krasheninnikova Yu.S., Tolpin V.A., Maklakov S.M., Savchenko E.V., Capabilities of hyperspectral indices analysis of the Vega-Constellation remote monitoring information systems, Sovremennye problemy distantsionnogo zondirovaniya Zemli iz kosmosa, 2016, 13(3), pp. 28-45. (In Russian).

[13] Rouse J.W., Haas R.H., Scheel J.A., Deering D.W., Monitoring Vegetation Systems in the Great Plains with ERTS, Proceedings, 3rd Earth Resource Technology Satellite (ERTS) Symposium, 1974, 1, pp. 48-62.

[14] Savorskiy V.P., Panova O.Yu., Savchenko E.V., Danilychev M.V., Kashnitskiy A.V., Kutuza I.B., Machikhin A.S., Specialized spectral indices for identifying areas of illegal plant cultivation, ARMIMP-2018 Akustoopticheskiye $i$ radiolokatsionnyye metody izmereniy $i$ obrabotki informatsii: Materialy $11-y$ Mezhdunarodnoy nauchno-tekhnicheskoy konferentsii. Rossiyskoye NTORES im. A.S. Popova. Suzdal', 2018, pp. 86-90. (In Russian). 